\title{
PET response assessment in apatinib-treated radioactive iodine-refractory thyroid cancer
}

\author{
Chen Wang ${ }^{1,2, *}$, Xin Zhang ${ }^{1,2, *}$, Xue Yang ${ }^{3}$, Hui Li1,2, Ruixue Cuil,2, Wenmin Guan4, Xin Li5, Zhaohui Zhu1,2 and \\ Yansong Lin1,2
}

1Department of Nuclear Medicine, Peking Union Medical College Hospital, Beijing, China

2Beijing Key Laboratory of Molecular Targeted Diagnosis and Therapy in Nuclear Medicine, Beijing, China

3Department of Oncology, The Affiliated Hospital of Qingdao University, Qingdao, China

${ }^{4}$ Department of Radiology, Peking Union Medical College Hospital, Beijing, China

5Department of Nuclear Medicine, Zhejiang Cancer Hospital, Hangzhou, China

Correspondence should be addressed to Y Lin: linys@pumch.cn

*(C Wang and $\mathrm{X}$ Zhang contributed equally to this work)

\begin{abstract}
This work evaluated the use of the positron emission tomography (PET)/computed tomography (CT) technique to assess the early therapeutic response and predict the prognosis of patients with radioactive iodine-refractory differentiated thyroid cancer (RAIR-DTC) who underwent apatinib therapy. Standardised uptake value (SUV), metabolic tumour volume (MTV) and total lesion glycolysis (TLG), derived from ${ }^{18 F-F D G ~ P E T / C T ~ a n d ~}$ SUV from ${ }^{68} \mathrm{Ga}-\mathrm{NOTA}-\mathrm{PRGD} 2 \mathrm{PET} / \mathrm{CT}$ were evaluated. Tumour response was evaluated using the Response Evaluation Criteria in Solid Tumors (RECIST) 1.1. Sixteen of 20 patients achieved partial response (PR) and four of 20 had stable disease (SD) after apatinib therapy. Six progression-free survival (PFS) events occurred. A strong correlation was observed between the best change in the sum of the longest diameters of target lesions $(\triangle \mathrm{CT} \%)$ and ${ }^{18} \mathrm{~F}-\mathrm{FDG} \mathrm{PET} / \mathrm{CT}$ indices after the completion of the first treatment cycle ( $\triangle \mathrm{MTV} \%(P=0.0019), \Delta \mathrm{TLG} \%(P=0.0021)$ and $\triangle \mathrm{SUVmax} \%(P=0.0443))$. A significant difference in PFS was observed between patients with $\triangle \mathrm{MTV} \%<-45 \%$ and $\geq-45 \%$ $(P=0.0019)$ and between patients with $\triangle$ TLG $\%<-80 \%$ and $\geq-80 \%(P=0.0065)$. Ten of 11 patients presented a decrease in SUVmax on ${ }^{68} \mathrm{Ga}$-NOTA-PRGD2 PET/CT after two cycles of apatinib therapy and showed PR, whereas one patient presenting an increase in SUVmax only showed SD as the best response. When a cut-off value of the target/background ratio at $-20 \%$ was used, two PFS curves showed a significant difference $(P=0.0016)$. Hence, early assessment by ${ }^{18}$ F-FDG and ${ }^{68}$ Ga-NOTA-PRGD2 PET/CT was effective in the prediction and evaluation of RAIR-DTC treated with apatinib.
\end{abstract}

\author{
Key Words \\ - radioactive iodine-refractory \\ thyroid cancer \\ - apatinib \\ - PET \\ - integrin receptor $a_{v} \beta_{3}$ \\ imaging \\ - response assessment \\ - BRAFV600E \\ - TERT promoter mutation
}

\section{Introduction}

Recent decades have witnessed a rapid increase in the incidence of differentiated thyroid cancer (DTC) worldwide (Chen et al. 2016, Siegel et al. 2016). Arising from aberrant follicular cells, DTC accounts for nearly
95\% of all thyroid neoplasms (Brose et al. 2014). Most patients with DTC achieve favourable outcomes with surgery, radioactive iodine (RAI) therapy and thyroxine therapy; however, up to $10 \%$ of patients with metastatic 
disease are eventually found to have radioactive iodinerefractory DTC (RAIR-DTC) (Schlumberger et al. 2015). Because of the low survival rate and limited therapeutic regimen, the treatment of RAIR-DTC has become a hot topic in the recent years (Ho et al. 2013, Brose et al. 2014, Schlumberger et al. 2015, Yang et al. 2017). With the development of mechanisms of vascular endothelial growth factor (VEGF) and its receptors (VEGFR), a series of tyrosine kinase inhibitors (TKIs) that target VEGF/VEGFR pathway have been proven effective against RAIR-DTC (Schlumberger et al. 2015, Lin et al. 2017). Traditionally, patients with RAIR-DTC were evaluated every 2 months after the therapy using the Response Evaluation Criteria in Solid Tumors (RECIST) based on conventional imaging technologies, such as computed tomography (CT). The identification of the biomarkers of an early therapeutic response to TKI therapy is of immense clinical relevance because it may help identify patients who are unlikely to benefit from the therapy. The rapid development of positron emission tomography (PET), PET/CT, new metabolic imaging and receptorbased imaging techniques has led to their use in clinical practice (Wahl et al. 2009). Studies have demonstrated the usefulness of $18 \mathrm{~F}$-fluorodeoxyglucose (18F-FDG) PET/CT for early response evaluation and prognosis assessment (Carr et al. 2010, Takahashi et al. 2012, Nakajo et al. 2017). Recently, except standardised uptake value (SUV), ${ }^{18}$ F-FDG PET/CT parameters, such as metabolic tumour volume (MTV) and total lesion glycolysis (TLG), have been shown to be of much clinical relevance in solid tumours such as oesophageal and pancreatic adenocarcinoma (Sakane et al. 2017, van Rossum et al. 2017). However, little has been reported for DTC, particularly for RAIR-DTC, after TKI therapy.

Among the integrin family, the integrin receptor $\mathrm{a}_{\mathrm{v}} \beta_{3}$ is one of the most important molecules that participate in tumour angiogenesis, invasion and metastasis (Zheng et al. 2015, Cheng et al. 2016). As a small peptide, arginine-glycine-aspartic acid (RGD) may selectively bind to $\mathrm{a}_{\mathrm{v}} \beta_{3}$. This lock-key relationship allows the use of radionuclide labelled RGD to localise the tumour and for neovascularisation. ${ }^{68} \mathrm{Ga}$-NOTA-PRGD2 has been used to detect lymph node metastases of lung cancer (Zheng et al. 2015). In our previous study, we employed 99mTc-3PRGD2 to localise RAIR-DTC lesions (Zhao et al. 2012). Furthermore, previous studies have demonstrated a correlation between integrin $\mathrm{a}_{\mathrm{v}} \beta_{3}$ and VEGFR (Ehling et al. 2016, Helal-Neto et al. 2016). Zhu et al. have used RGD to evaluate synovial angiogenesis in patients with rheumatoid arthritis (Zhu et al. 2014). On the basis of previous studies, it would be reasonable to use RGD as a predictor of efficacy and prognosis after TKI therapy targeting the VEGF/VEGFR pathway.

Apatinib, a novel small-molecule TKI that selectively targets VEGFR-2, has been shown to inhibit VEGF-mediated endothelial cell migration and proliferation and decrease tumour microvascular density (Ding et al. 2013). Our previous study has shown a quick response and encouraging efficacy of apatinib in RAIRDTC (Lin et al. 2017). Therefore, in this study, we used PET to identify the early response and predict the prognosis of RAIR-DTC treated with apatinib.

\section{Materials and methods}

This study was approved by the Institutional Review Board of Peking Medical College Hospital Ethics Committee and was conducted in accordance with the Declaration of Helsinki and Good Clinical Practice guidelines. Written informed consent was obtained from all patients prior to their enrolment. This study was part of the prospective phase II clinical trial registered with ClinicalTrails.gov (number, NCT02731352).

\section{Patients}

Patients aged $\geq 18$ years with histologically confirmed DTC were recruited. The inclusion criteria were patients who qualified the definition of RAIR-DTC; at least one progressive lesion according to RECIST (version 1.1) and disease progression within the past 14 months according to RECIST (version 1.1). Patients who qualified in at least one of the following criteria were defined as RAIR-DTC: (1) presence of at least one measurable lesion without iodine uptake on radioactive iodine scan; (2) presence of at least one measurable lesion that had progressed within the past 12 months even if it showed positive uptake of radioactive iodine or (3) received cumulative activity of ${ }^{131} \mathrm{I} \geq 600 \mathrm{mCi}$.

\section{Study design and treatment regimen}

Twenty patients were sequentially recruited for apatinib therapy (Jiangsu Hengrui Medicine Co., Ltd, Lianyungang, China). Initially, the first 10 patients were treated with apatinib at an initial dose of $750 \mathrm{mg}$ based on a previous phase I study (Li et al. 2010) and a pharmacokinetics study (Ding et al. 2013). Despite encouraging efficacy, a high incidence of Grade 3 adverse events (AEs) was observed within 8 weeks of initiation of the treatment; 
consequently, the dose was reduced to $500 \mathrm{mg}$ for the 10 subsequently enrolled patients. A treatment cycle was defined as 4 weeks (28 days). Treatment interruption due to the toxicity of apatinib was allowed for no more than 2 times or for a maximum duration of 2 weeks (either continuously or cumulatively) in one cycle. Dose reduction by $250 \mathrm{mg}$ q.d. each time was accepted, and re-escalation was not accepted. Treatment was continued until disease progression, drug intolerance or the withdrawal of consent for participation in the study. The study diagram is shown in Fig. 1.

The primary endpoints of this phase II clinical trial were the change in serum $\mathrm{Tg}$ concentration, disease control rate (DCR) and objective response rate (ORR), and the secondary endpoints were overall survival, progression-free survival (PFS) and duration of response. All patients were required to undergo ${ }^{18} \mathrm{~F}$-fluorodeoxyglucose (18F-FDG) PET/CT scan thrice (at baseline and at the completion of 1st and 2nd treatment cycles). ${ }^{68} \mathrm{Ga}-\mathrm{NOTA}-\mathrm{PRGD} 2$ PET/CT scan twice (at baseline and after two cycles of treatment) was recommended but not mandatory. The present study aimed to analyse whether PET can be used to assess the early therapeutic response and predict the prognosis of RAIR-DTC treated with apatinib, regardless of the dosage.

\section{Positron emission tomography imaging}

18F-FDG PET/CT scan was conducted to assess the response to apatinib. Briefly, patients were instructed to avoid strenuous work or exercise for at least 1 day and fast for $>4 \mathrm{~h}$ before the ${ }^{18} \mathrm{~F}-\mathrm{FDG}(0.15 \mathrm{mCi} / \mathrm{kg})$ injection. After injection, patients rested in a warm, dark room for 40-60 min.

${ }^{68} \mathrm{Ga}$-NOTA-PRGD2 was synthesised using the method described elsewhere (Zheng et al. 2015). The radiochemical purity of the product ${ }^{68} \mathrm{Ga}-\mathrm{NOTA}-\mathrm{PRGD} 2$ exceeded $95 \%$. After intravenous injection of $111-185 \mathrm{MBq}$ (3-5 mCi) of ${ }^{68} \mathrm{Ga}$-NOTA-PRGD2, patients were asked to rest for nearly $40 \mathrm{~min}$. The PET acquisitions were performed $1 \mathrm{~h}$ after the injection.

The acquisition was performed from skull to midthigh (five to six beds position, 2 min per bed) using a Siemens Biograph 64 Truepoint TrueV PET/CT scanner.

The PET/CT data sets were reconstructed using iterative reconstruction (ordered subset expectation maximum, OSEM) and attenuation correction. The results were measured using the software on the PET/CT system's workstation (TrueD, Siemens Medical Systems).

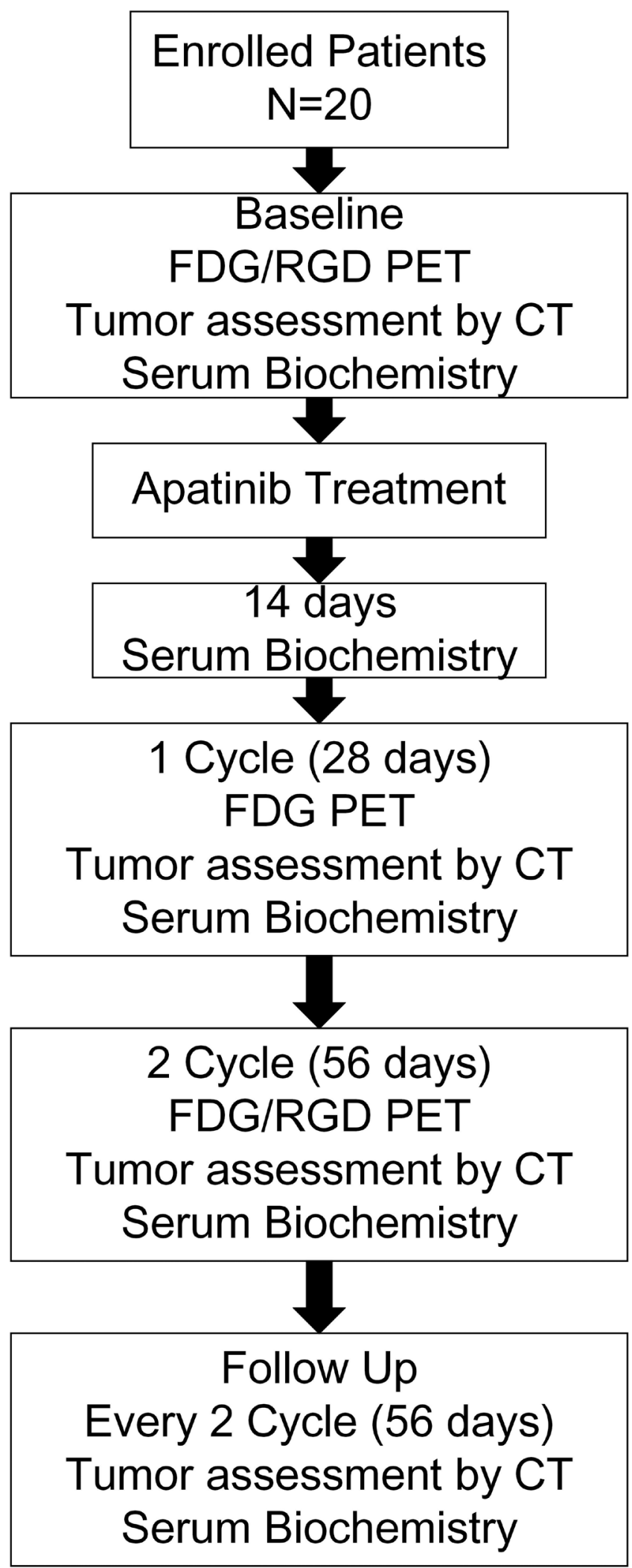

Figure 1

Study design. 
Positron emission tomography image interpretation and metabolic parameter measurement

All PET images were interpreted consensually by two nuclear medicine physicians, who were blinded to the clinical data. Semi-quantitative analysis was performed on the PET/CT system's workstation. Target lesions according to RECIST 1.1 were selected at baseline and evaluated during follow-up. The primary tumour was defined as the volume of interest (VOI) and delineated on PET scans using an in-built semi-automatic 3D method.

SUV was derived using the following expression: (decay-corrected administered activity in kilobecquerels per millilitre of tissue volume)/(injected FDG activity in kilobecquerels/body weight in grams). MTV was derived by applying a threshold of $40 \%$ of the maximum standardised uptake value (SUVmax) as the lowest limit of the segmentation criteria. The computer-assisted automatically derived contouring margins and regions of interest for both measurements were checked on three sectional images (axial, coronal and sagittal) to ensure the accurate inclusion of all involved nodal sites and the exclusion of adjacent normal structures. TLG was calculated as SUVmean $\times$ MTV.

The following quantitative parameters were extracted from VOIs of the ${ }^{18} \mathrm{~F}-\mathrm{FDG}$ PET/CT scan: maximum and mean SUV (SUVmax and SUVmean), MTV and TLG. For ${ }^{68} \mathrm{Ga}$-NOTA-PRGD2 PET/CT, only SUVmax and SUVmean were evaluated. Furthermore, the target lesions/background ratios (T/B, SUVmax of target lesions/SUVmean of background) were calculated for further analysis. $\triangle \mathrm{SUV} \%$ was calculated using (SUV-SUV baseline $/ \mathrm{SUV}_{\text {baseline, }}$ as well as $\Delta \mathrm{MTV} \%$ and $\triangle \mathrm{TLG} \%$.

\section{${ }^{18} \mathrm{~F}-\mathrm{FDG}$ positron emission tomography response evaluation}

The ${ }^{18} \mathrm{~F}-\mathrm{FDG}$ response was evaluated using the European Organization for Research and Treatment of Cancer (EORTC) PET study group criteria. Complete metabolic response was achieved when the SUVs of all lesions decreased to the level of uptake equivalent in the background tissue. Partial metabolic response (PMR) was defined as a reduction of a minimum of $25 \%$ of the sum of SUVmax of target lesions. Stable metabolic disease (SMD) was classified as an increase in tumour sum of SUVmax less than $25 \%$ or decrease less than $25 \%$, and no visible increase in the extent of tumour uptake. Progressive metabolic disease (PMD) was defined as an increase of the sum of SUVmax $>25 \%$ or visible increase in the extent of 18 F-FDG uptake ( $>20 \%$ in the longest dimension) or when new ${ }^{18}$ F-FDG uptake appeared in metastatic lesions.

\section{Assessment of biochemical and clinical response}

Serum biochemical tests were performed at baseline and every two weeks during the initial two cycles and every 4 weeks after the second cycle. The tests included $\mathrm{Tg}$, thyroglobulin antibody (TgAb) and thyroid-stimulating hormone (TSH).

Tumour diameters were measured at baseline, after one cycle and every two cycles thereafter using CT. The tumour response was evaluated as defined by RECIST (version 1.1). The percentage change in sum of tumour diameters $(\Delta \mathrm{CT} \%)$ was calculated by (diameter-diameter baseline $_{\text {e }}$ / diameter baseline $_{\text {. }}$

PFS was defined as the time from initial drug administration until disease progression or death, whichever occurred first. DCR was defined as complete response (CR), partial response (PR) or stable disease (SD); ORR was defined as CR and PR according to RECIST (version 1.1).

AEs were recorded and assessed at baseline until 4 weeks after the last drug administration in the study.

\section{Measurement of $\mathrm{Tg}, \mathrm{TgAb}$ and TSH}

$\mathrm{Tg}$ and $\mathrm{TgAb}$ levels were determined using the electrochemiluminescence immunoassay (Roche Diagnostics $\mathrm{GmbH}$ ) with functional sensitivity values of $0.100 \mathrm{ng} / \mathrm{mL}$ and $10 \mathrm{IU} / \mathrm{mL}$, respectively. TSH was determined using the chemiluminescence immunoassay (Siemens Healthcare Diagnostics), with a measuring range from 0.004 to $150 \mu \mathrm{IU} / \mathrm{mL}$. TgAb values $>100 \mathrm{IU} / \mathrm{mL}$ were considered positive. $\Delta \mathrm{Tg} \%$ was calculated as $\left(\mathrm{Tg}-\mathrm{Tg}_{\text {baseline }}\right) / \operatorname{Tg}_{\text {baseline }}$.

\section{BRAFV600E and TERT promoter mutation analysis}

The primary tumour lesions of thyroid were used for the DNA analysis. Genomic DNA was extracted and analysed using the protocol reported previously (Yang et al. 2017).

\section{Statistical analysis}

The predictive accuracy of the changes in the four metabolic parameters (SUVmax, SUVmean, MTV and TLG) for tumour response was evaluated using receiveroperating characteristic (ROC) analysis; the area under the 
curve (AUC) was used as an index of accuracy. Optimal cut-off values were derived from the ROC curves aiming for the best sensitivity and specificity. PFS curve was estimated using Kaplan-Meier analysis and compared using the log-rank (Mantel-Cox) test; the median and values around the median reduction in the four metabolic parameters were used to find the optimal cut-off values for PFS prediction analysis.

Categorical variables are expressed as frequencies and percentage; continuous variables are expressed as mean \pm standard deviation (s.D.) or median and interquartile range when appropriate. A two-sample $t$-test or Wilcoxon rank-sum test was used to assess betweengroup differences. Correlation between two variables was analysed using Pearson or Spearman test according to the data. SPSS (version 19.0; SPSS) and Prism 6 (GraphPad Software) were used for statistical analyses. $P<0.05$ was considered statistically significant.

\section{Results}

\section{Patients}

A total of 20 patients were enrolled in this study. The mean age of patients was $54.7 \pm 13.3$ (range, 27-78) years; the male-to-female ratio was 1:1. All patients underwent total or near-total thyroidectomy and lymph node (LN) resection, and the diagnosis of DTC was confirmed on histopathological examination. Fifteen of 20 patients lost the ability of RAI uptake in the metastatic lesions at the first RAI treatment after successful remnant ablation. The baseline characteristics of the included patients are summarised in Table 1. Five patients experienced failure of pre-TKI therapy; of these, four patients were treated with sorafenib and one patient was treated with both sorafenib and vandetanib. All patients underwent 18F-FDG PET/CT scans on three occasions, while 11 patients underwent ${ }^{68} \mathrm{Ga}$-NOTA-PRGD2 PET/CT on two occasions. Nine patients presented pulmonary metastases only, one patient presented bone metastases only and one patient presented mediastinal LN metastases. The other nine patients presented both pulmonary and bone $(N=5)$ or head $(N=2)$ or neck $(N=2)$ metastases (Table 1$)$.

\section{Efficacy}

\section{The RECIST response to apatinib}

During apatinib therapy, 16 (80\%) patients achieved PR and four (20\%) patients achieved SD, according to RECIST 1.1 (Table 1). The DCR and ORR of the study were $100 \%$
$(20 / 20)$ and $80 \%(16 / 20)$, respectively. After apatinib therapy, the sum of the longest diameters of target lesions decreased from $37.1 \pm 15.5$ to $20.7 \pm 9.0 \mathrm{~cm}$. The best change in the sum of the longest diameters of target lesions from baseline $(\Delta \mathrm{CT} \%)$ in all patients was $-42.2 \%$ (range, $-0.9 \%$ to $-73.2 \%$; Fig. $2 \mathrm{~A}$ ).

From March 2016 to August 2017, six PFS events occurred, which included five disease progressions and one death. A female patient died of pulmonary infection after 5.53 months of therapy. Until she died, her target lesion response was still PR according to CT. Another patient quit the clinical trial after 6 months and showed $\mathrm{SD}$ at the end of the treatment. The estimated median PFS for patients with endpoints $(N=6)$ was 6.5 months. The other 13 patients were still under follow-up with a treatment time range from 7.5 to 17.5 months.

\section{Change in serum Tg levels}

$\mathrm{Tg}$ levels were available for 15 out of the 20 patients. Five patients were excluded from the analyses; among these, three patients presented positive TgAb as previously described, and the other two patients showed quite low levels of $\mathrm{Tg}(0.43$ and $0.36 \mathrm{ng} / \mathrm{mL}$ at baseline, respectively).

The mean $\mathrm{Tg}$ dropped from 2455.9 (range, $0.36-13,808) \mathrm{ng} / \mathrm{mL}$ to 101.2 (range, 396.1-0.1) ng/mL after apatinib therapy (Fig. 2B).

For the $15 \mathrm{Tg}$-eligible patients, the $\Delta \mathrm{Tg} \%$ at 2-week $\left(\Delta \operatorname{Tg}_{2 \mathrm{w}} \%\right)$ and one cycle $\left(\Delta \operatorname{Tg}_{1 \text { cycle }} \%\right)$ and the lowest $\Delta \operatorname{Tg} \%$ were calculated. The $\Delta \operatorname{Tg}_{\text {lowest }} \%$ ranged from $-33.2 \%$ to $-98.5 \%, \Delta \operatorname{Tg}_{2 \mathrm{w}} \%$ from $+47.4 \%$ to $-91.3 \%$ and $\Delta \operatorname{Tg}_{1 \text { cycle }} \%$ from $+38.7 \%$ to $-95.2 \%$. Four patients showed an interim increase in Tg at 2 weeks or one cycle, followed by decrease during follow-up.

\section{PET findings}

\section{F-FDG PET/CT scan}

For one-cycle assessment, 16 PMR, three SMD and one PMD were detected according to the EORTC criteria. For two-cycle assessment, one patient was excluded as the target lesion was not amenable to evaluation because of the influence of distinct uptake of the heart. As a result, 14 patients achieved PMR and five had SMD.

At baseline, the initial mean sum of SUVmax of FDG uptake in target lesions was 12.27 (median, 8.38; range, 1.59-42.41). After one cycle of therapy, the mean SUVmax decreased to 5.41 (median, 4.26; range, 1.30-27.73). The mean SUVmax after two cycles of therapy was 4.51 (median, 4.36; range, 0.32-9.67). The other parameters also 
Table 1 Characteristics of patients.

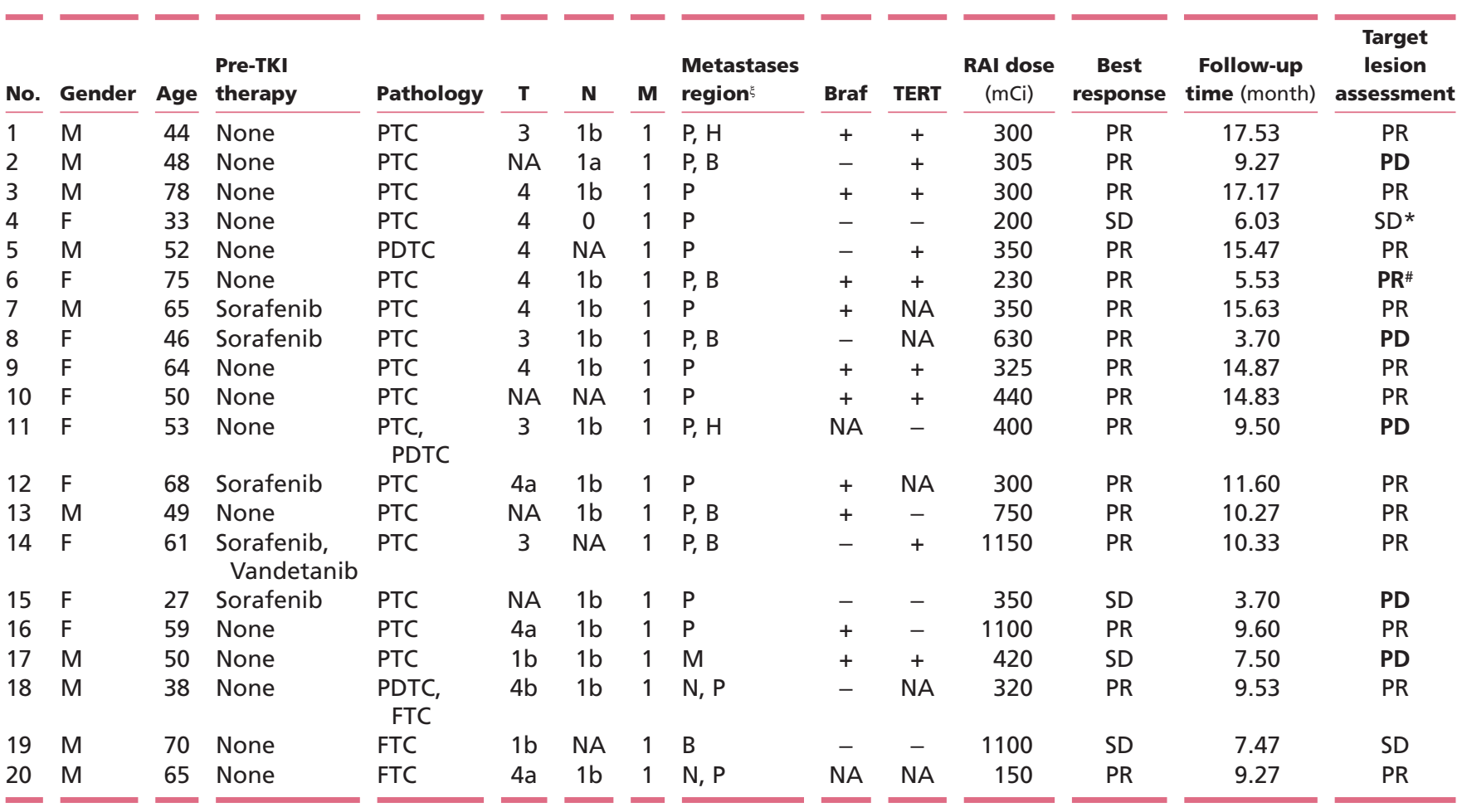

EP, pulmonary; $\mathrm{H}$, head; $\mathrm{B}$, bone; $\mathrm{M}$, mediastinum lymph node; $\mathrm{N}$, neck lymph node; *this patient withdrew the consent; \#this patient died of pulmonary infection. Bold indicates that PFS event occurred in these patients.

FTC, follicular thyroid cancer; NA, not available; PDTC, poorly differentiated thyroid cancer; PTC, papillary thyroid cancer.

exhibited a similar trend (mean SUVmean from 4.37 to 3.42 and 2.87; MTV from 10.87 to 5.73 and 4.78; TLG from 52.68 to 19.73 and 13.02 , respectively) (Fig. 2C and D).

\section{Correlation and predictive role of PET finding to morphologic responses}

No significant correlation or agreement was observed between RECIST 1.1 and EORTC criteria (Spearman $P=0.217$, Kappa $P=0.197$ ).

A strong correlation was observed between the best $\triangle \mathrm{CT} \%$ and $\Delta \mathrm{MTV} \%$ (Spearman $r \quad 0.6511, P=0.0019$ ), $\Delta$ TLG\% (Spearman $r$ 0.6451, $P=0.0021$ ) and $\Delta$ SUVmax\% (Spearman $r 0.4541, P=0.0443$ ) at one-cycle treatment. Correlation of $\triangle \mathrm{MTV} \%$ (Spearman $r$ 0.7034, $P=0.0008$ ) and $\Delta$ TLG\% (Spearman $r 0.7896, P<0.0001$ ) with $\Delta$ CT\% was also significant in two-cycle PET/CT scan (Fig. 3).

Patients were divided into two groups according to the best response (RECIST 1.1): 16 patients in the PR group and three in the SD group (one patient excluded because of the influence of heart uptake as described earlier). The parameters were compared between the two groups. A significant between-group difference was observed with respect to $\Delta \mathrm{MTV} \%$ and $\Delta \mathrm{TLG} \%$, at the completion of the first and second treatment cycles (Mann-Whitney $U$ test, $P=0.019 ; P=0.044, P=0.019$ and $P=0.014$, respectively).

ROC curve showed that $\triangle \mathrm{MTV} \%$ and $\Delta \mathrm{TLG} \%$ at onecycle had a great value in predicting PR response (AUC: 0.859 and 0.797 , respectively). The optimal cut-off values of $\Delta \mathrm{MTV} \%$ and $\triangle \mathrm{TLG} \%$ at one-cycle $(-20.78 \%$ and $-56.34 \%$, respectively) were associated with sensitivity values of 0.75 and 0.75 , respectively, and specificity values of 0.94 and 0.81 , respectively. In contrast, $\Delta$ SUVmax $\%$ and $\Delta$ SUVmean $\%$ did not show any significant predictive value for PR response.

\section{Comparative analyses between biochemistry and imaging responses}

All the 15 patients eligible for the $\mathrm{Tg}$ analysis showed a significant decrease in the sum of the longest diameters of target lesions. The best $\Delta \mathrm{CT} \%$ ranged from $-18.6 \%$ to $-73.2 \%$. For PET response at one cycle, $\triangle \mathrm{SUVmax} \%$ ranged from $75.6 \%$ to $-82.7 \%, \Delta \mathrm{MTV} \%$ from $-13.8 \%$ to $-86.4 \%$ and $\Delta \mathrm{TLG} \%$ from $-19.5 \%$ to $-95.4 \%$. For the four patients who showed a temporary increase in $\mathrm{Tg}$, the sum of the longest diameters of target lesions all showed shrinkage after apatinib therapy. Further correlation 


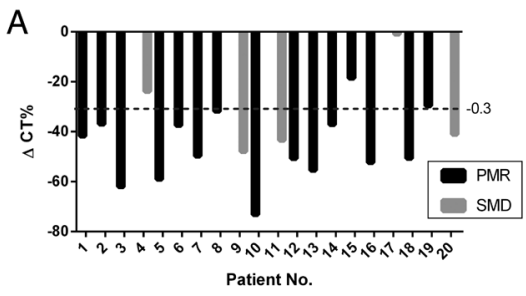

C

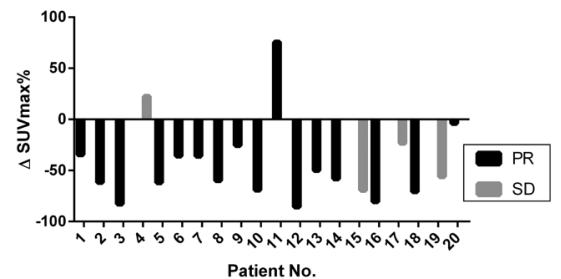

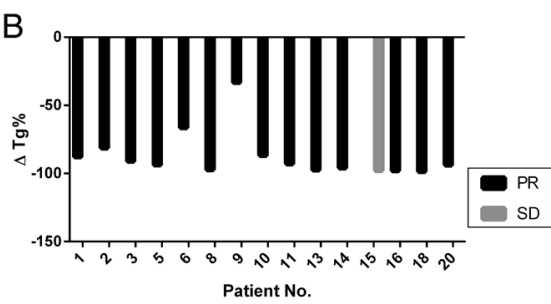

D

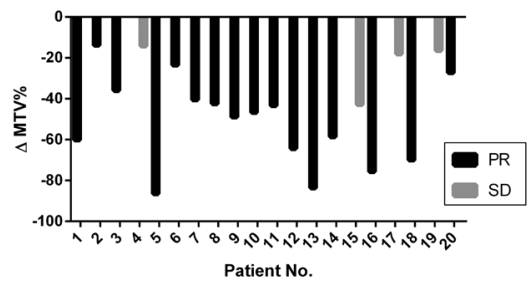

\section{Figure 2}

Clinical response after apatinib therapy. (A) The best change in the sum of the longest diameters of target lesions ( $\triangle \mathrm{CT} \%$ ) according to RECIST 1.1 . (B) The best change in Tg level after apatinib therapy. (C) Change in SUVmax after one cycle of apatinib therapy. (D) Change in MTV after one cycle of apatinib therapy. PMR, partial metabolic response; $\mathrm{PR}$, partial response; $\mathrm{SD}$, stable disease; SMD, stable metabolic disease. analyses (Spearman test) showed no significant correlation between the $\Delta \mathrm{Tg} \%$ (the best, 2 weeks and one cycle) and $\Delta \mathrm{CT} \%$ (the best, one cycle and two cycles), $\Delta$ SUVmax $\%$, $\Delta \mathrm{MTV} \%$ and $\Delta \mathrm{TLG} \%$ at one cycle, respectively. The results indicate no linear relationship between biochemistry and imaging response (for the statistical value and $P$ values, Supplementary Table 1 , see section on supplementary data given at the end of this article).

For the 15 patients, only one patient had SD, and the other 14 patients achieved PR as the best response. For the SD patient, a decrease in Tg level was also observed with the best $\Delta \mathrm{Tg} \%$ of $-98 \%$. The Tg decrease seemed to have no predictive value for the response.

\section{PFS according to FDG PET findings}

The parameters of ${ }^{18}$ F-FDG PET were used in the analyses. For $\triangle \mathrm{SUVmax} \%$ and $\triangle \mathrm{SUVmean} \%$ at one cycle or two cycles, the use of a cut-off value of $-25 \%$ (EORTC criteria) failed to show a significant difference in PFS. Fortunately, both $\triangle \mathrm{MTV} \%$ and $\triangle \mathrm{TLG} \%$ showed promising results. When a cut-off value of $-45 \%$ was used for $\Delta \mathrm{MTV} \%$ at one cycle, a significant difference was observed between
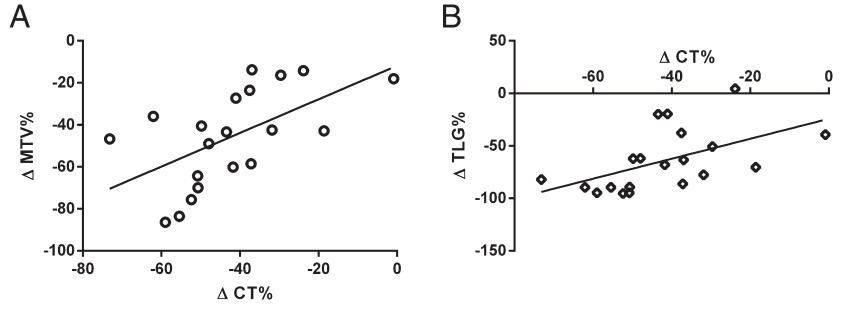

Figure 3

Correlation between MTV (A), TLG (B) change after one cycle of therapy and the best of the sum of the longest diameters of target lesions $(\triangle C T \%)$. MTV, metabolic tumour volume; TLG, total lesion glycolysis. the two PFS curves $(P=0.0019)(\geq-45 \%, N=11$, median PFS 9.3 months (95\% CI, 7.0-11.6); $<-45 \% N=9$, median PFS not achieved) (Fig. 4A), as well as $\triangle \mathrm{MTV} \%$ at 2-cycle $(P=0.0001)(\geq-45 \%, N=7$, median PFS 7.5 months $(95 \%$ CI, 4.0-11.0); <-45\% $N=12$, median PFS not achieved). Regarding $\triangle \mathrm{TLG} \%$, when a cut-off value of $-80 \%$ was used, significant differences were also observed at 1-cycle $(P=0.0065)(\geq-80 \%, N=12$, median PFS 9.5 months $(95 \%$ CI, 6.8-12.2); $<-80 \% N=12$, median PFS not achieved; Fig. 4B) and 2-cycle $(P=0.0029)(\geq-80 \%, N=10$, median PFS 9.3 months (95\% CI, 7.0-11.6), $<-80 \% N=9$, median PFS not achieved).

\section{${ }^{68 G a-N O T A-P R G D 2 ~ P E T / C T ~ s c a n ~}$}

Eleven patients underwent ${ }^{68} \mathrm{Ga}-\mathrm{NOTA}-\mathrm{PRGD} 2 \mathrm{PET} / \mathrm{CT}$ scan. At baseline, the mean sum of SUVmax was 4.42 (range 0.97-8.69). After two treatment cycles, the mean sum of SUVmax decreased to 1.48 (range 0.22-2.18). There was no significant correlation between the reduction in RGD $\Delta$ SUVmax $\%$ and FDG $\triangle \mathrm{SUVmax} \%, \Delta \mathrm{MTV} \%$, $\Delta \mathrm{TLG} \%$, the best $\Delta \mathrm{CT} \%$ or two-cycle $\Delta \mathrm{CT} \%$. A total of 10 patients showed a decrease in SUVmax of ${ }^{68} \mathrm{Ga}-N O T A-$ PRGD2 after apatinib therapy and showed PR as the best
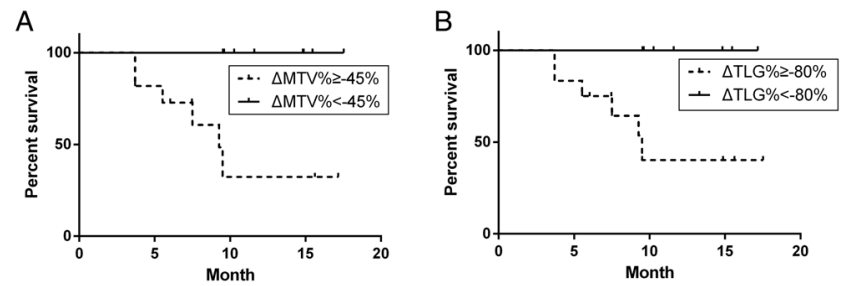

Figure 4

Progression-free survival (PFS) according to different $\triangle \mathrm{MTV} \%$ (A) and $\triangle T L G \%$ of one-cycle therapy. MTV, metabolic tumour volume; TLG, total lesion glycolysis. (c) 2018 Society for Endocrinology Published by Bioscientifica Ltd. Printed in Great Britain 
A

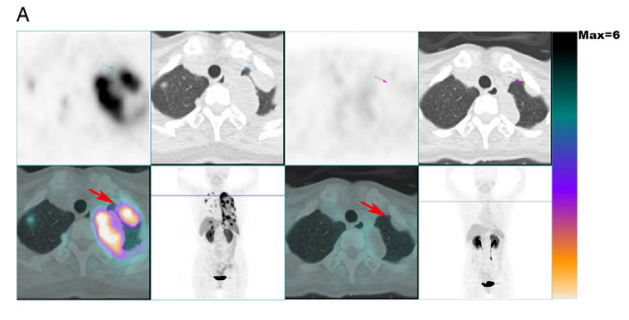

B

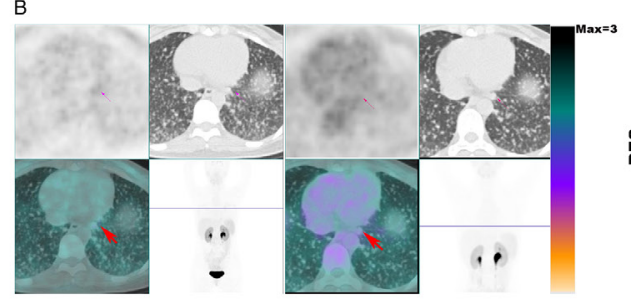

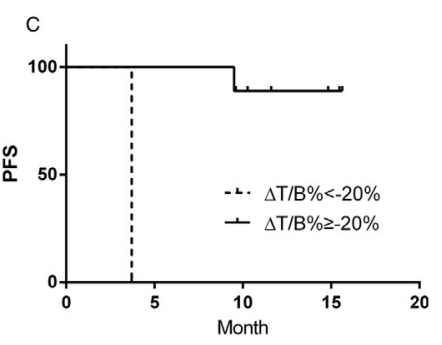

Figure 5

The PET findings ${ }^{68}$ Ga-NOTA-PRGD2 PET/CT Patient no. 14 (A) and 15 (B) before (left) and after (right) two cycles of apatinib therapy. (A) After two cycles of therapy, this patient showed a significant decrease in the uptake (SUVmax from 6.532 to 0.74 ) and presented partial response (PR) after the therapy (still PR after 10.3 months follow-up). (B) Patient showed an increase in the uptake (SUVmax from 0.975 to 1.239 ) and showed progression disease at the two-cycle (3.7 months) assessment. (C) Progress-free survival according to the ${ }^{68}$ Ga-NOTA-PRGD2 PET findings.

response (Fig. 5A). One patient who showed an increase in SUVmax of ${ }^{68} \mathrm{Ga}-\mathrm{NOTA}-\mathrm{PRGD} 2$ after two treatment cycles only had SD as the best response (Fig. 5B). We further used target/background ratio (T/B, SUVmax of target lesions/SUVmean of background) for analysis. The mean T/B ratio decreased from 4.43 (range 1.10-10.04) to 1.86 (range $1.08-3.30$ ). Nine patients presented a notable decrease in $\mathrm{T} / \mathrm{B}$ ratio $(\Delta \mathrm{T} / \mathrm{B} \%)$ after therapy (from $-39.4 \%$ to $-75.2 \%)$. Furthermore, one patient showed a limited change in $\mathrm{T} / \mathrm{B}$ after therapy $(-0.16 \%)$ and the other one showed an increase in $\mathrm{T} / \mathrm{B}$ after apatinib therapy $(+19.5 \%)$. These two patients showed PD at four-cycle assessment. When a cut-off value of T/B ratio at $-20 \%$ was used, the two survival curves showed a significant difference $(P=0.0016 ;$ Fig. 5C). Hence, ${ }^{68} \mathrm{Ga}$-NOTA-PRGD2 PET/CT may potentially be used not only as a response assessment tool but also as a means to predict post-treatment PFS.

\section{$B R A F$ and TERT promoter mutation}

In total, two and five patients were not available for the detection of BRAFV600E and TERT promoter mutations, respectively. Ten of the 18 patients harboured BRAFV600E mutation, whereas eight tested negative. Nine of 15 patients tested positive for TERT promoter mutation, whereas six tested negative.

Out of the four patients who showed SD, one harboured BRAFV600E mutation. In the PR group, nine of 14 harboured BRAF mutation. There was no significant difference between the two groups $(P=0.2745)$. Eight of nine patients with TERT mutation achieved PR, whereas three of six in the TERT-negative group achieved PR; no significant between-group difference was observed with respect to $P R$ rate $(P=0.2352)$.

No significant difference was observed with respect to PFS between the BRAF mutation-positive and BRAF mutation-negative subgroups $(P=0.3039)$ and between the TERT mutation-positive and TERT mutation-negative groups $(P=0.7481)$.

\section{Safety}

Most patients experienced treatment-related AEs, ranging from Grade 1-3 AEs; no severe AEs (SAE) related to apatinib were observed till data collection. The most common AEs were hand-foot skin reaction, proteinuria, increased AST levels, fatigue and hypertension. The details are shown in Supplementary Table 1.

\section{Discussion}

Recent years have witnessed rapid advances in the field of molecular targeted therapies including TKI therapy for thyroid cancer (Ferrara et al. 2003, Xing et al. 2013). Thus far, FDA has approved sorafenib and lenvatinib for the treatment of RAIR-DTC (Brose et al. 2014, Schlumberger et al. 2015). Furthermore, a recent study has reported encouraging response rates and efficacy of apatinib (a novel TKI targeting VEGFR-2) in RAIR-DTC after 2 months of treatment (Lin et al. 2017). In this study, although all patients did not achieve PR with apatinib therapy, the PR rate was still very high (16/20 patients). Out of the five patients who failed to respond to pre-TKI (sorafenib or vandetanib), four showed PR after apatinib therapy and experienced prolonged PFS. These findings are encouraging and suggest the role of apatinib as a salvage treatment after first-line TKI therapy. Nevertheless, not all patients benefited much from apatinib therapy. Therefore, the early prediction of tumour response and prognosis is of great importance for the optimisation of the management of patients on TKI therapy because of the multiple side effects and uncertain efficacy. Therefore, we conducted this study to identify the factors that may http://erc.endocrinology-journals.org https://doi.org/10.1530/ERC-18-0007 (c) 2018 Society for Endocrinology Published by Bioscientifica Ltd. Printed in Great Britain 
help predict the response to and the outcomes of TKI therapy in patients with RAIR.

Functional imaging modalities, such as PET, represent an invaluable clinical tool. ${ }^{18} \mathrm{~F}-\mathrm{FDG}$ PET/CT parameters, such as MTV and TLG, have been shown to predict the therapeutic response in patients with oesophageal cancer (Nakajo et al. 2017). However, their role in thyroid cancer (particularly for RAIR-DTC) is not well characterised. To the best of our knowledge, our work may be the first research focusing on the ${ }^{18} \mathrm{~F}-\mathrm{FDG}$ PET/CT parameters in RAIR-DTC. Both $\triangle \mathrm{MTV} \%$ and $\triangle \mathrm{TLG} \%$ showed encouraging values in predicting response and prognosis compared with $\Delta$ SUVmax $\%$ and $\Delta$ SUVmean $\%$. Parameters such as MTV and TLG reflect the metabolic status of the entire tumour and are potentially more accurate for tumour characterisation than is SUVmax, which only reveals a single voxel among the tumour region. In our study, $\triangle \mathrm{MTV} \%$ and $\triangle \mathrm{TLG} \%$ were found valuable in two aspects. First, because of the strong correlation between the best $\Delta \mathrm{CT} \%$ and $\Delta \mathrm{MTV} \%$ (Spearman $r$ 0.6511, $P=0.0019$ ) and between the best $\Delta \mathrm{CT} \%$ and $\Delta \mathrm{TLG} \%$ (Spearman $r$ 0.6451, $P=0.0021$ ), clinicians may use $\Delta \mathrm{MTV} \%$ and $\Delta \mathrm{TLG} \%$ at the first cycle to predict the shrinkage of tumour lesions. Furthermore, the extent of $\Delta \mathrm{MTV} \%$ and $\triangle \mathrm{TLG} \%$ also showed a significant difference between the PR and SD groups $(P=0.019, P=0.044, P=0.019$ and $P=0.014$, respectively). ROC analyses also demonstrated the usefulness of $\triangle \mathrm{MTV} \%$ and $\triangle \mathrm{TLG} \%$ in predicting PR. This provides clinicians a convenient tool for the early prediction and evaluation, which may help avoid unnecessary TKI therapy. Second, we found that both $\triangle \mathrm{MTV} \%$ and $\triangle \mathrm{TLG} \%$ showed a correlation with PFS. As one of the most important parameter for cancer patients, PFS may reflect the patient's benefit and prognosis. The ability to predict disease progression at an early stage is of vital importance because it may facilitate any change in the treatment regimen and optimise patient management strategy. Consequently, both clinicians and radiologists may use ${ }^{18} \mathrm{~F}-\mathrm{FDG}$ PET/CT to predict the prognosis of patients undergoing TKI therapy during the early stages. This may help avoid the unnecessary treatment of patients who are unlikely to benefit from TKI and to timely identify the PD status.

The best time for early PET is unclear. Takahashi et al. conducted PET scan 2 days after gefitinib therapy in patients with lung cancer (Takahashi et al. 2012). Wang et al. performed PET 14 days after chemotherapy for gastric cancer. Carr et al. conducted PET scan 7 days after sunitinib therapy for thyroid cancer but failed to achieve any significant result (Carr et al. 2010). It is widely known that due to the inflammatory reaction induced by chemotherapy for lymphoma, it is recommended that ${ }^{18}$ F-FDG PET in patients with lymphoma should be performed 3 weeks after the completion of chemotherapy (Barrington et al. 2014). Although it is unclear whether TKI can result in the same consequences, we opted for PET scan at one cycle (28 days) after TKI therapy.

In this work, we also used ${ }^{68} \mathrm{Ga}$-NOTA-PRGD2 PET/CT as an evaluator and predictor, probably the earliest work in the thyroid field. Previous study used 99mTc-3PRGD2 to detect and locate the RAIR lesions while simultaneously revealing the uptake of RGD in lesions of RAIR-DTC. We further used ${ }^{68} \mathrm{Ga}$-labelled RGD and PET imaging for preand post-treatment evaluation of uptake at the target lesions. The uptake of RGD may reflect neovascularisation as it selectively binds to $a_{v} \beta_{3}$ expressed in neo-vascular areas in tumour tissues. Like all of the anti-angiogenesis drugs, apatinib targets VEGFR-2 and exerts its anti-tumour effect by blocking the VEGF/VEGFR waterfall signal pathway and inhibiting neovascularisation. Till now, there has been no specific tool to predict the prognosis after TKI therapy targeting VEGF/VEGFR pathway. Bao et al. used ${ }^{18} \mathrm{~F}$-Alfatide II to monitor the early response in U87MG tumour mice (Bao et al. 2016). Hence, it would be a useful tool if RGD imaging could reveal the response and prognosis in patients treated with TKI. In this work, ${ }^{68}$ Ga-NOTA-PRGD2 was not only effective in evaluation of response, but was also found to be a potential predictor of prognosis after TKI therapy. According to our results, two patients with an insignificant decrease in RGD uptake (revealed by $\Delta \mathrm{T} / \mathrm{B} \%$ ) showed $\mathrm{PD}$ after two cycles of apatinib therapy. Despite the small sample size, ${ }^{68} \mathrm{Ga}$-NOTA-PRGD2 may prove to be a useful tool for response evaluation and prognostic assessment of patients treated with TKI.

In recent years, advances in molecular pathology have played a key role in unravelling the pathogenesis of cancers, such as the role of BRAFV600E and TERT promoter in thyroid cancer (Xing 2013, Xing et al. 2013, 2014). Xing et al. reported the pejorative survival of thyroid patients harbouring BRAFV600E Or TERT mutation (Xing et al. 2013, 2014). Yang et al. also found that Chinese patients with TERT mutation were refractory to radioactive iodine therapy (Yang et al. 2017). Initial work also showed a relationship between BRAFV600E mutation and dedifferentiation of thyroid cancer (Xing 2007). Similarly, patients harbouring both BRAFV600E and TERT mutation were refractory to radioactive iodine, which may result in unfavourable prognosis (Yang et al. 2017). Previous studies have reported the prognosis of patients 
with BRAFV600E mutation after TKI therapy (Brose et al. 2014, Tahara et al. 2017); in both placebo- and sorafenib/ lenvatinib-treated groups, patients with the BRAFV600E mutation showed a better response than those with wildtype BRAF. Moreover, PFS benefit was maintained in patients harbouring the BRAFV600E mutation. However, the BRAF mutation status was not predictive of TKI benefit according to the biomarker-treatment interaction analysis. Regarding TERT promoter mutation, not much evidence is available for patients with RAIR treated with TKI. Our study included 14 patients with BRAF ${ }^{V 600 E}$ or TERT promoter mutation. We found no significant association of BRAFV600E or TERT promoter mutation status with the therapeutic response or PFS, which may suggest that TKI therapy improves the unfavourable prognoses of patients harbouring the BRAFV600E or TERT promoter mutation under the natural course of the disease.

As the foremost biomarker of DTC, Tg revealed tumour burden and treatment response. Yang et al. reported the practical utility of Tg to predict post-RAI therapy prognosis using the response system of DTC (Yang et al. 2016). As Tg is produced both by normal thyroid tissues and DTC cells, it may reflect the tumour burden in patients with DTC, whereas $\mathrm{Tg}$ level in patients with RAIR-DTC varied from 0.36 to $13,808 \mathrm{ng} / \mathrm{mL}$ in our study. The two patients with low $\mathrm{Tg}$ levels $(0.36$ and $0.43 \mathrm{ng} / \mathrm{mL})$ harboured a large tumour burden. We believe that in this circumstance, since the tumour was undergoing dedifferentiation and was refractory to iodine, Tg might not be a sensitive marker of tumour burden or response as compared to that in patients with well-differentiated DTC. Despite the fact that $\mathrm{Tg}$ levels in all patients showed a decreasing trend after apatinib therapy, the $\Delta \mathrm{Tg} \%$ level showed no correlation with imaging findings. Furthermore, four patients showed temporary increase in $\mathrm{Tg}$ after apatinib therapy at two or four weeks, followed by a decrease during follow-up. This temporary increase in Tg level might be due to treatmentinduced injury and death of tumour cells. Thus, we think that $\mathrm{Tg}$ had nothing to do with prognosis but may be of value in predicting response.

In summary, early assessment by ${ }^{18 F-F D G ~ a n d ~}$ ${ }^{68} \mathrm{Ga}$-NOTA-PRGD2 PET/CT may help in prognostic assessment of patients with RAIR-DTC treated with apatinib. Reductions in MTV and TLG were both useful for the prediction of the response and PFS. ${ }^{68} \mathrm{Ga}-\mathrm{NOTA}-\mathrm{PRGD} 2$ might be a potential tool for the evaluation of RAIR-DTC treated with TKI. Both BRAFV600E and TERT promoter mutations failed to show an obvious influence on the response and PFS after apatinib therapy. Considering the small sample size in this study, these encouraging results will be verified in a phase III clinical trial (ClinicalTrails. gov, NCT03048877).

\section{Supplementary data}

This is linked to the online version of the paper at https://doi.org/10.1530/ ERC-18-0007.

\section{Declaration of interest}

The authors declare that there is no conflict of interest that could be perceived as prejudicing the impartiality of the research reported.

\section{Funding}

This work was supported by National Natural Science Foundation of China (Grant No. 81571714), CSCO-Hengrui Research Foundation (No. Y-HR2016032), and Innovation Funds for Postgraduates of Peking Union Medical College Hospital (No. 1002-02-08). The funders had no role in study design, data collection and analysis, decision to publish, or preparation of the manuscript.

\section{Acknowledgments}

The authors thank the patients and their families as well as all investigators participated in this study. They also thank Jiangsu Hengrui Medicine Co., Ltd for providing apatinib. Furthermore, they would also like to thank Enago (www.enago.com/bioscientifica) for the English language editing.

\section{References}

Bao X, Wang MW, Luo JM, Wang SY, Zhang YP \& Zhang YJ 2016 Optimization of early response monitoring and prediction of cancer antiangiogenesis therapy via noninvasive PET molecular imaging strategies of multifactorial bioparameters. Theranostics 6 2084-2098. (https://doi.org/10.7150/thno.13917)

Barrington SF, Mikhaeel NG, Kostakoglu L, Meignan M, Hutchings M, Mueller SP, Schwartz LH, Zucca E, Fisher RI, Trotman J, et al. 2014 Role of imaging in the staging and response assessment of lymphoma: consensus of the International Conference on Malignant Lymphomas Imaging Working Group. Journal of Clinical Oncology 32 3048-3058. (https://doi.org/10.1200/JCO.2013.53.5229)

Brose MS, Nutting CM, Jarzab B, Elisei R, Siena S, Bastholt L, de la Fouchardiere C, Pacini F, Paschke R, Shong YK, et al. 2014 Sorafenib in radioactive iodine-refractory, locally advanced or metastatic differentiated thyroid cancer: a randomised, double-blind, phase 3 trial. Lancet 384 319-328. (https://doi.org/10.1016/S01406736(14)60421-9)

Carr LL, Mankoff DA, Goulart BH, Eaton KD, Capell PT, Kell EM, Bauman JE \& Martins RG 2010 Phase II study of daily sunitinib in FDG-PET-positive, iodine-refractory differentiated thyroid cancer and metastatic medullary carcinoma of the thyroid with functional imaging correlation. Clinical Cancer Research 16 5260-5268. (https:// doi.org/10.1158/1078-0432.CCR-10-0994)

Chen W, Zheng R, Baade PD, Zhang S, Zeng H, Bray F, Jemal A, Yu XQ $\&$ He J 2016 Cancer statistics in China, 2015. CA: A Cancer Journal for Clinicians 66 115-132. (https://doi.org/10.3322/caac.21338)

Cheng W, Feng F, Ma C \& Wang H 2016 The effect of antagonizing RGD-binding integrin activity in papillary thyroid cancer cell lines. OncoTargets and Therapy 9 1415-1423. (https://doi.org/10.2147/OTT. S99166) 
Ding J, Chen X, Gao Z, Dai X, Li L, Xie C, Jiang H, Zhang L \& Zhong D 2013 Metabolism and pharmacokinetics of novel selective vascular endothelial growth factor receptor-2 inhibitor apatinib in humans. Drug Metabolism and Disposition 41 1195-1210. (https://doi. org/10.1124/dmd.112.050310)

Ehling J, Misiewicz M, von Stillfried S, Mockel D, Bzyl J, Pochon S, Lederle W, Knuechel R, Lammers T, Palmowski M, et al. 2016 In situ validation of VEGFR-2 and alpha v ss 3 integrin as targets for breast lesion characterization. Angiogenesis 19 245-254. (https://doi. org/10.1007/s10456-016-9499-4)

Ferrara N, Gerber HP \& LeCouter J 2003 The biology of VEGF and its receptors. Nature Medicine 9 669-676. (https://doi.org/10.1038/ nm0603-669)

Helal-Neto E, Brandao-Costa RM, Saldanha-Gama R, Ribeiro-Pereira C, Midlej V, Benchimol M, Morandi V \& Barja-Fidalgo C 2016 Priming endothelial cells with a melanoma-derived extracellular matrix triggers the activation of alphavbeta3/VEGFR2 axis. Journal of Cellular Physiology 231 2464-2473. (https://doi.org/10.1002/jcp.25358)

Ho AL, Grewal RK, Leboeuf R, Sherman EJ, Pfister DG, Deandreis D, Pentlow KS, Zanzonico PB, Haque S, Gavane S, et al. 2013 Selumetinib-enhanced radioiodine uptake in advanced thyroid cancer. New England Journal of Medicine 368 623-632. (https://doi. org/10.1056/NEJMoa1209288)

Li J, Zhao X, Chen L, Guo H, Lv F, Jia K, Yv K, Wang F, Li C, Qian J, et al. 2010 Safety and pharmacokinetics of novel selective vascular endothelial growth factor receptor-2 inhibitor YN968D1 in patients with advanced malignancies. BMC Cancer 10 529. (https://doi. org/10.1186/1471-2407-10-529)

Lin Y, Wang C, Gao W, Cui R \& Liang J 2017 Overwhelming rapid metabolic and structural response to apatinib in radioiodine refractory differentiated thyroid cancer. Oncotarget $842252-42261$. (https://doi.org/10.18632/oncotarget.15036)

Nakajo M, Jinguji M, Nakabeppu Y, Nakajo M, Higashi R, Fukukura Y, Sasaki K, Uchikado Y, Natsugoe S \& Yoshiura T 2017 Texture analysis of (18)F-FDG PET/CT to predict tumour response and prognosis of patients with esophageal cancer treated by chemoradiotherapy. European Journal of Nuclear Medicine and Molecular Imaging $\mathbf{4 4}$ 206-214. (https://doi.org/10.1007/s00259-016-3506-2)

Sakane M, Tatsumi M, Hori M, Onishi H, Tsuboyama T, Nakamoto A, Ota T, Eguchi H, Wakasa K, Hatazawa J, et al. 2017 Volumetric parameters of 2-deoxy-2-[18F]fluoro-d-glucose positron emission tomography/computed tomography can predict histopathologic treatment response after neoadjuvant chemoradiotherapy in pancreatic adenocarcinoma. European Journal of Radiology 94 64-69. (https://doi.org/10.1016/j.ejrad.2017.05.021)

Schlumberger M, Tahara M, Wirth LJ, Robinson B, Brose MS, Elisei R, Habra MA, Newbold K, Shah MH, Hoff AO, et al. 2015 Lenvatinib versus placebo in radioiodine-refractory thyroid cancer. New England Journal of Medicine 372 621-630. (https://doi.org/10.1056/ NEJMoa1406470)

Siegel RL, Miller KD \& Jemal A 2016 Cancer statistics, 2016. CA: A Cancer Journal for Clinicians 66 7-30. (https://doi.org/10.3322/ caac.21332)

Tahara M, Schlumberger M, Elisei R, Habra MA, Kiyota N, Paschke R, Dutcus CE, Hihara T, McGrath S, Matijevic M, et al. 2017 Exploratory analysis of biomarkers associated with clinical outcomes from the study of lenvatinib in differentiated cancer of the thyroid. European Journal of Cancer 75 213-221. (https://doi.org/10.1016/j. ejca.2017.01.013)

Takahashi R, Hirata H, Tachibana I, Shimosegawa E, Inoue A, Nagatomo I, Takeda Y, Kida H, Goya S, Kijima T, et al. 2012 Early [18F]fluorodeoxyglucose positron emission tomography at two days of gefitinib treatment predicts clinical outcome in patients with adenocarcinoma of the lung. Clinical Cancer Research 18 220-228. (https://doi.org/10.1158/1078-0432.CCR-11-0868)

van Rossum PS, Fried DV, Zhang L, Hofstetter WL, Ho L, Meijer GJ, Carter BW, Court LE \& Lin SH 2017 The value of (18)F-FDG PET before and after induction chemotherapy for the early prediction of a poor pathologic response to subsequent preoperative chemoradiotherapy in oesophageal adenocarcinoma. European Journal of Nuclear Medicine and Molecular Imaging 44 71-80. (https://doi. org/10.1007/s00259-016-3478-2)

Wahl RL, Jacene H, Kasamon Y \& Lodge MA 2009 From RECIST to PERCIST: Evolving Considerations for PET response criteria in solid tumors. Journal of Nuclear Medicine 50 (Supplement 1) 122S-150S. (https://doi.org/10.2967/jnumed.108.057307)

Xing M 2007 BRAF mutation in papillary thyroid cancer: pathogenic role, molecular bases, and clinical implications. Endocrine Reviews 28 742-762. (https://doi.org/10.1210/er.2007-0007)

Xing M 2013 Molecular pathogenesis and mechanisms of thyroid cancer. Nature Reviews Cancer 13 184-199. (https://doi.org/10.1038/ $\operatorname{nrc} 3431)$

Xing M, Alzahrani AS, Carson KA, Viola D, Elisei R, Bendlova B, Yip L, Mian C, Vianello F, Tuttle RM, et al. 2013 Association between BRAF V600E mutation and mortality in patients with papillary thyroid cancer. JAMA 309 1493-1501. (https://doi:10.1001/jama.2013.3190)

Xing M, Liu R, Liu X, Murugan AK, Zhu G, Zeiger MA, Pai S \& Bishop J 2014 BRAF V600E and TERT promoter mutations cooperatively identify the most aggressive papillary thyroid cancer with highest recurrence. Journal of Clinical Oncology 32 2718-2726. (https://doi. org/10.1200/JCO.2014.55.5094)

Yang X, Liang J, Li T, Zhao T \& Lin Y 2016 Preablative stimulated thyroglobulin correlates to new therapy response system in differentiated thyroid cancer. Journal of Clinical Endocrinology and Metabolism 101 1307-1313. (https://doi.org/10.1210/jc.2015-4016)

Yang X, Li J, Li X, Liang Z, Gao W, Liang J, Cheng S \& Lin Y 2017 TERT promoter mutation predicts radioiodine-refractory character in distant metastatic differentiated thyroid cancer. Journal of Nuclear Medicine 58 258-265. (https://doi.org/10.2967/jnumed.116.180240)

Zhao D, Jin X, Li F, Liang J \& Lin Y 2012 Integrin alphavbeta3 imaging of radioactive iodine-refractory thyroid cancer using $99 \mathrm{mTc}$ 3PRGD2. Journal of Nuclear Medicine 53 1872-1877. (https://doi. org/10.2967/jnumed.112.107821)

Zheng K, Liang N, Zhang J, Lang L, Zhang W, Li S, Zhao J, Niu G, Li F, Zhu Z, et al. 2015 68Ga-NOTA-PRGD2 PET/CT for integrin imaging in patients with lung cancer. Journal of Nuclear Medicine $\mathbf{5 6}$ 1823-1827. (https://doi.org/10.2967/jnumed.115.160648)

Zhu Z, Yin Y, Zheng K, Li F, Chen X, Zhang F \& Zhang X 2014. Evaluation of synovial angiogenesis in patients with rheumatoid arthritis using ${ }^{68} \mathrm{Ga}$-PRGD2 PET/CT: a prospective proof-of-concept cohort study. Annals of the Rheumatic Diseases 73 1269-1272. (https://doi.org/10.1136/annrheumdis-2013-204820)

Received in final form 20 March 2018

Accepted 28 March 2018

Accepted Preprint published online 4 April 2018 (c) 2018 Society for Endocrinology Published by Bioscientifica Ltd. Printed in Great Britain 